\title{
LES CONDITIONS DE PRODUCTION DU DISCOURS POLITIQUE
}

\begin{abstract}
Christian Le Bart ${ }^{1}$
Les conditions de production du discours politique ont changé en France comme dans toutes les démocraties occidentales. La contrainte d'exemplarité, qui obligeait les professionnels de la politique à se conformer aux logiques institutionnelles, est désormais critiquée pour sa lourdeur et sa prévisibilité. Les politiques peuvent désormais jouer la carte de la singularité, de la spontanéité, voire de l'improvisation. Plus précisément, ils doivent alterner entre ces deux registres (raideur institutionnelle et relâchement) selon qu'ils évoluent en contexte institutionnel ou bien en contexte plus médiatique.
\end{abstract}

L'exemplarité linguistique a longtemps été valorisée comme forme d'excellence du discours politique. Elle mesurait l'adéquation au rôle du locuteur : il fallait bien parler et s'ajuster au rôle institutionnel endossé. La médiatisation de la vie politique conduit aujourd'hui à davantage valoriser la distance au rôle, l'improvisation, voire le relâchement. Selon les situations et les publics, les locuteurs jouent tantôt la carte de l'exemplarité, tantôt celle du relâchement.

L'analyse du discours politique se déploie traditionnellement dans deux directions différentes. La première, interne, prend le discours comme objet et tente de le faire parler en donnant à voir des réalités

1 Christian Le Bart est Professeur de science politique à Sciences Po Rennes.

Recherches en communication, $\mathrm{n}^{\circ} 41$ (2014). 
discursives dont on peut dire qu'elles ne sont pas visibles à l'œil nu (ou audibles à l'oreille nue), c'est-à-dire dans les conditions ordinaires de la réception médiatique. L'exemple le plus convainquant pour illustrer cette démarche est l'analyse lexicométrique : le travail de quantification permet de dégager, par comparaison entre corpus, des reliefs significatifs (Mayaffre, 2004). La seconde démarche se situe du côté de la sociologie, de l'histoire et de la science politique. Elle consiste en l'analyse des conditions de production du discours politique (Le Bart, 1998). Elle est donc, à la différence de la précédente, externe. L'objectif est ici de recenser toutes les variables qui pèsent sur un locuteur et qui l'amènent à dire ce qu'il dit (et pas autre chose), et à le dire d'une façon particulière (et pas autrement). Les orientations de recherche sont multiples : on peut s'intéresser au locuteur en tant que personne (quelle est sa trajectoire sociale et politique ? Quel rôle occupe-t-il ? Quelles stratégies poursuit-il en l'instant $t$ où il s'exprime ? Quels sont les éléments constitutifs de son identité qui sont à même de transparaître dans son propos ou dans son style ?...) ; on peut élargir la focale et s'intéresser au contexte de la situation de communication (quel ancrage institutionnel ? Quel public ? Quelle médiatisation? Quels événements antérieurs ou à venir? Quels enjeux ? Quel agenda politique ?...) et même plus largement encore au contexte sociopolitique au sein duquel prend place l'interaction en question (régime et situation politiques, calendrier électoral, situation nationale et internationale...). La difficulté est évidemment de parvenir à faire tenir ensemble toutes ces variables : ainsi verra-t-on dans la plus ponctuelle allocution d'un ministre à la fois le produit immédiat d'une intentionnalité tactique (exister dans le champ politique, s'imposer au sein d'un gouvernement, convaincre un public-cible, se rappeler au bon souvenir d'un électorat, s'imposer au sein d'un ministère...) et la résultante qui vient condenser une pluralité d'histoires entrelacées (histoire d'un individu, d'un ministère, histoire de la communication politique...).

Ces deux approches sont pertinentes et se complètent. Idéalement, il faudrait autant que possible les croiser, pour mesurer à partir de corpus précis les effets des diverses variables possibles. La revue francophone Mots (sous-titre : Les langages du politique) s'efforce depuis plusieurs décennies de mener à bien un tel projet ${ }^{1}$. L'entreprise est difficile et se heurte à des obstacles des deux côtés : l'analyse de discours tend

1 Le projet fut lancé en 1980 en particulier à l'initiative de Maurice Tournier. Le directeur en est aujourd'hui Paul Bacot. Pour une synthèse, voir le numéro anniversaire pour les trente ans de la revue (Mots, 2010). 
parfois à s'enfermer dans le matériau textuel et fait preuve de naïveté dans la mobilisation des variables explicatives ; symétriquement, la sociologie du discours méconnaît trop celui-ci et en reste trop souvent à la technique déproblématisée de la citation qui illustre à défaut de démontrer.

On ne prétendra évidemment pas ici dépasser ces faiblesses. Nous voudrions plus simplement formuler, du point de vue de la sociologie du discours politique, une hypothèse de recherche relative à l'évolution de celui-ci. Nous esquisserons, exemple français à l'appui, une comparaison entre le discours politique classique, celui des débuts de la Cinquième République, et le discours plus contemporain. Le premier est marqué par le principe de la vigilance discursive : il faut bien parler et faire sur ce terrain comme sur les autres preuves d'exemplarité (§1). Aujourd'hui au contraire, les politiques n'hésitent plus à prendre leur distance par rapport à la norme linguistique : ils osent des écarts pour faire vrai, pour faire authentique, pour faire spontané (\$2). Tout dépend évidemment des situations et des locuteurs. Il n'y a pas passage d'une façon de parler politique à une autre mais glissement progressif selon une logique qui est davantage celle de la sédimentation que celle de la rupture. En fonction des contextes, les professionnels de la politique apprennent à jouer tantôt la carte de l'exemplarité (bien parler), tantôt celle du parler relâché (§3).

Notre ambition est de fournir quelques clés permettant d'expliquer ces évolutions. Elles ne se situent évidemment pas à hauteur de locuteur : ce dernier est pris dans un jeu de déterminations qui encadrent et structurent les stratégies discursives qu'il développe. Nous raisonnerons à l'échelle macrosociologique, et nous invoquerons la crise des institutions politiques comme variable centrale. C'est elle qui permet de rendre compte de la moindre pertinence des stratégies d'exemplarité institutionnelle. A l'inverse, la médiatisation (et même la peopolisation) du champ politique incite les gouvernants à jouer la carte de la distance au rôle, de la spontanéité plutôt que celle de la prévisibilité. S'écarter de la norme, qu'elle soit linguistique ou autre (vestimentaire par exemple), peut être aujourd'hui la meilleure façon de gagner en légitimité auprès d'une opinion publique moins centrée que jadis sur l'exemplarité institutionnelle. 


\section{L'exemplarité institutionnelle du discours politique classique}

Les débuts de la Cinquième République sont marqués par la force des institutions sociales en général (famille, école, entreprise...) et politique en particulier (État, partis politiques). Dans ce contexte fort bien décrit par François Dubet (2002), la légitimité et la grandeur sont d'abord des ressources institutionnelles. Les individus peuvent en bénéficier à condition de s'appuyer sur elles : autorité et légitimité du professeur, du médecin, du maire ou du notable... Ceux-ci ont donc intérêt à coller au plus près des institutions, à représenter celles-ci avec docilité et déférence. Ainsi voit-on s'affirmer un intérêt à l'exemplarité : plus l'individu est à même de signifier l'institution, mieux il parvient à incarner cette dernière, plus il pourra recueillir confiance et légitimité. Ainsi de l'homme d'État ou du notable exemplaire, à qui l'on confiera d'autant plus volontiers le pouvoir de décider qu'ils semblent dignes d'incarner l'institution et le collectif. Être à la hauteur du rôle est un préalable à la légitimité.

On trouve trace de ce souci d'exemplarité sur tous les terrains constitutifs de la présentation de soi des politiques : mise en scène de la vie familiale, apparence physique et vestimentaire, et bien sûr pratiques discursives. S'agissant de cette question spécifiquement, on observera le souci partagé par tous les professionnels de la politique de bien parler. La langue légitime est celle de l'école et de l'université, les écarts sont des fautes, les gouvernants appartiennent à une élite qui doit être exemplaire. La langue française est à la fois considérée comme un élément central du patrimoine immatériel national et comme un ciment permettant l'activation d'un espace public également national. On attend donc des politiques qu'ils habitent cette langue, qu'ils la célèbrent, et donc aussi qu'ils la respectent.

La sociologie du recrutement politique ajuste quasi mécaniquement les élus à cette exigence. Souvent d'origine bourgeoise, plus souvent encore surdiplômés, les professionnels de la politique n'ont guère à se forcer pour bien parler. Il y a ajustement spontané, pour la plupart d'entre eux, entre les exigences du champ et l'habitus dont ils sont porteurs et qui les pousse spontanément, presque naturellement, vers la norme. Affleure parfois le souci de faire peuple (hypocorrection) mais dans des conditions peu institutionnelles (sur le terrain). La diversité des contextes et des publics oblige les professionnels de la politique à jouer d'une certaine diglossie : mais les contextes institutionnels les plus 
exposés appellent tout naturellement le respect de la norme linguistique. On peut se laisser aller sur les marchés ou en fin de réunion, on se tient à la tribune ou à la télévision. N'est-il pas alors acquis pour tout le monde que l'art de bien parler constitue une ressource essentielle pour toute carrière politique ? L'histoire de la République a consacré le modèle du parlementaire professionnel du discours (avocat ou journaliste), formé aux humanités, orateur avant d'être expert (Rousselier, 1997) ?

La force de la norme linguistique connaît toutefois deux types d'exception. La première renvoie par exemple aux écarts de langage d'un Georges Marchais, premier secrétaire du Parti communiste. A la différence de la plupart de ses concurrents, et à la différence même de la plupart des hauts responsables communistes comme Pierre Juquin ou Roland Leroy, Georges Marchais s'exprime dans une langue qui n'est pas irréprochable au regard de l'orthodoxie linguistique. Ses subjonctifs sont approximatifs, ses constructions grammaticales souvent hasardeuses. Jouant objectivement le rôle de défenseurs de la norme, les humoristes (par exemple Thierry Le Luron) ne cessent de brocarder son style, soulignant fortement l'illégitimité du personnage et l'incongruité de sa prétention à faire de la politique. Le plus intéressant tient sans doute à l'impossibilité objective pour le leader du PCF de retourner le stigmate ainsi activé. Il s'efforce certes de délégitimer les moqueries dont il est l'objet en invoquant la classe ouvrière dont il se veut le porte-parole : ne pas être allé à l'école peut devenir, dans la logique de représentation des milieux populaires qui est celle du PC, un atout sinon un titre de gloire. Mais force est de reconnaître que ces stratégies ouvriéristes sont peu efficaces. Tout se passe comme si, selon le modèle alors théorisé par Pierre Bourdieu et Jean-Claude Passeron (1970), la culture académique bénéficiait en France d'une légitimité absolue. Elle seule est recevable, et il ne saurait être question pour un politique de prétendre s'en émanciper : ne pas la posséder est une faute, une insuffisance, la marque d'un déficit. Le PC ne participe-t-il pas, d'ailleurs, de cette reconnaissance, en se dotant de porte-parole atypiquement pourvus en ressources culturelles et plus armés que la plupart des camarades pour jouer le jeu bourgeois de la politique ? Rien ne permet évidemment de savoir si les approximations linguistiques de Georges Marchais ont participé de son leadership partisan (effets internes à l'organisation partisane) ou si celui-ci s'est développé en dépit de ce handicap. Il est en revanche acquis que la postérité a consacré Georges Marchais en animal politique d'envergure exceptionnelle. Il est un des premiers à attirer l'attention par ces écarts-mêmes. Il anticipe 
d'une certaine façon le modèle contemporain du politique médiatique qui fait le spectacle, qui surprend.

L'autre exception à laquelle on ne peut pas ne pas penser se situe au contraire au sommet du champ politique. Le général de Gaulle s'autorise des formules linguistiquement osées mais qui font politiquement mouche par la seule position de leur auteur. Le charisme du général n'est pas un attribut de sa personne : il caractérise un rapport de force qui impose par exemple à ses interlocuteurs la posture seconde de faire-valoir ou d'exégète. Les bons mots restés fameux disent sans doute le talent d'un homme d'esprit ; ils disent surtout la réussite d'une entreprise politique visant à placer le titulaire de la fonction présidentielle au-dessus des normes régissant ordinairement le champ politique. Il y a de ce point de vue homologie stricte entre la position institutionnelle conférée au président par la constitution de 1958 (et de 1962) et ce que l'on pourrait appeler sa position discursive : il peut prendre des libertés par rapport aux normes qui régissent le discours institutionnel comme il peut en prendre par rapport aux normes qui régissent la politique. Clé de voûte des institutions, le président est davantage force instituant que force instituée. Sa pratique fait jurisprudence davantage qu'elle n'obéit à la norme. Homme de lettres et homme du 18 juin, il impose une œuvre qui fait forcément violence à ce qui la précède.

La trivialité de certaines formules utilisées par le général ${ }^{1}$ bouscule le discours institutionnel, mais elle n'est pas condamnée car elle participe d'un leadership supposé charismatique. On crédite le général d'un style, quand les hommes d'État qui l'entourent doivent servir en silence. Le droit de se distinguer individuellement est très inégalement réparti dans le champ politique. Il est un privilège présidentiel. Pour les autres, à commencer par ses premiers ministres, la norme est l'exemplarité la plus impersonnelle.

\section{La dénonciation de la langue de bois et la valorisation de l'improvisation}

Un glissement s'est opéré depuis cette époque. L'excellence et l'exemplarité ne constituent plus des sources exclusives de légitimité. La télévision et plus généralement la médiatisation de la vie politique ont imposé les lois du spectacle jusque dans le champ politique. Adossé aux institutions, celui-ci l'est aussi, et de plus en plus, aux médias. Il en

1 Par exemple : « La réforme, oui, la chienlit, non » (19 mai 1968). 
résulte des changements significatifs au niveau des façons de faire de la politique. Quand prévalait jadis l'exemplarité du bien parler, s'impose désormais la recherche de singularité à tout prix : l'écart est valorisé.

Le premier terrain sur lequel on peut s'appuyer pour illustrer ce point est relatif à la prévisibilité du discours politique. Les institutions valorisent cette prévisibilité, elle est synonyme de fiabilité, elle marque l'emprise de l'institution sur l'individu, elle symbolise la continuité institutionnelle, voire l'immortalité des institutions. Car du point de vue des logiques institutionnelles, l'individu-locuteur est à la fois une ressource et une prise de risque : c'est certes grâce à lui que l'institution parvient à s'exprimer (l'individu met au service de l'institution sa faculté de parole et plus généralement son corps). Mais cette confiance placée dans l'individu comporte un risque : que celui-ci échappe à l'institution, qu'il se serve d'elle au lieu de la servir, qu'il n'abuse du droit de parole qu'on lui aura concédé. On sait comment, en pratique, les institutions limitent ce risque : elles socialisent au plus près les individus qu'elles prennent en charge, elles sélectionnent sévèrement ceux à qui reviendra le privilège de parler en leur nom, elles récompensent largement la docilité et la fiabilité.

La crise des institutions (Dubet, 2002) a grippé ces logiques de délégation. Au regard des canons médiatiques, la prévisibilité du porteparole est devenue un handicap. Encombré de ceux qu'il représente, le porte-parole apparait " lourd ", " langue-de-bois », ennuyeux, soporifique... Les médias valorisent à l'inverse le locuteur libre, qui sait faire la preuve qu'il est émancipé des institutions auxquelles il est attaché. Ainsi consacrent-ils volontiers comme personnalités politiques ceux que le champ politique aurait tendance à condamner comme " électrons libres »: ainsi des professionnels de la politique passant d'un camp à l'autre (Éric Besson, Bernard Kouchner...) ou n'hésitant pas à se distinguer par des prises de position hétérodoxes au sein de leur formation politique (Roselyne Bachelot à droite, Manuel Valls à gauche) ; ainsi encore des politiques jouant la stratégie du scandale en multipliant les provocations (Jean-Marie Le Pen); ainsi enfin des «petits entrepreneurs politiques » ayant créé leur propre formation politique pour s'émanciper des rigidités partisanes (François Bayrou, Jean-Luc Mélenchon, Philippe de Villiers, Nicolas Dupont-Aignan, Jean-Pierre Chevènement...)... Peu soucieux de fiabilité institutionnelle, toutes ces personnalités politiques mettent en avant la nécessité de s'exprimer avec franchise, de dire tout haut ce qui se dit tout bas, quitte à encourir les foudres des institutions qu'ils bousculent. Ces stratégies 
n'ont pourtant rien de sacrificielles : si l'implantation institutionnelle de leurs auteurs s'en trouve amoindrie, leur visibilité médiatique augmente en proportion.

Le refus de sacrifier à la langue de bois institutionnelle se double du refus de s'enfermer dans un usage appliqué de la norme linguistique standardisée. L'idée selon laquelle un élu de la République ne saurait impunément prendre des libertés avec le français académique n'a plus rien d'évidente. On voit ainsi se développer ce que l'on pourrait appeler des stratégies de relâchement qui témoignent d'une modification significative du rapport de force entre l'individu et son rôle (ou entre l'individu et l'institution qui lui a confié un rôle). L'exemple le plus probant est évidemment celui de Nicolas Sarkozy : quand ses devanciers demeuraient tenus par une exemplarité qui parfois induisait une certaine impersonnalité (Pompidou, VGE, Mitterrand et Chirac avaient surtout à cœur d'être à la hauteur du rôle construit par le général de Gaulle), lui n'hésite pas à développer un discours oral très familier. Le droit au style que l'on a évoqué s'agissant du général de Gaulle ne fut décliné et approprié par ses successeurs que sous la forme d'une excellence qui réconciliait exemplarité et singularité : le président devait parler mieux que les autres, il devait respecter scrupuleusement les normes linguistiques. La postérité a ainsi reconnu certaines formules qui participent du mythe du politique orateur capable d'improviser la formule qui fait mouche ( « vous n'avez pas le monopole du cœur $»^{1}$ ). On trouve peu d'exemples d'affranchissement brutal par rapport aux normes linguistique : le fameux " abracadabrantesque » de Jacques Chirac (20 septembre 2000) n'était-il pas emprunté à Rimbaud? Quant au verlan de François Mitterrand, il n'est exhibé face au journaliste Yves Mourousi que sur le mode du clin d'œil un rien canaille. Le président se risque, en 1985, à formuler deux ou trois mots («bléca », " chébran ») comme signe de la connaissance qu'il a de la société française (et, en l'occurrence, des jeunes de banlieue). Mais cette très ponctuelle référence fonctionne comme citation : le verlan n'est pas la langue du président. Il le connaît (ne connaît-il pas toute chose ?) mais ne le parle pas.

C'est sur ce point que la rupture sarkozyenne est la plus manifeste. Jouant la carte du naturel et de la spontanéité, celui-ci s'affranchit volontiers du rôle qui est le sien et des contraintes qui en principe

1 Valéry Giscard d'Estaing à l'occasion du débat télévisé face à François Mitterrand (10 mai 1974). 
l'encadrent. La médiatisation du " casse-toi pauv' con » (23 février 2008) démontre que ce glissement n'allait pas du tout de soi. On peut même défendre la thèse d'un désajustement au rôle qui aurait participé de la disgrâce du président UMP. Mais il conviendrait de ne pas oublier que ce style fait de spontanéité et de distance au rôle fut sans doute aussi une des clés du succès du même Nicolas Sarkozy (Charaudeau, 2008). Sur un mode moins outrancier que l'épisode précédent, il faut rappeler la propension du candidat puis du président à négliger le «ne » de la négation (« ch'sais pas »), à raccourcir les énoncés (" ch'uis » pour « je suis »), à utiliser le tutoiement, à désigner ses partenaires par leur prénom... Le style est relâché, la tension institutionnelle est ignorée, la frontière privé-public bousculée. La place de l'oralité est affirmée aux dépens de l'écrit, nouveau signe de la supériorité conférée à l'individu sur l'institution. Quand l'homme d'État traditionnel parle comme on écrit (au risque d'une lenteur dans l'élocution qui agace les médias), Nicolas Sarkozy privilégie l'oral. Il signe des ouvrages sans ambition littéraire chez des éditeurs sans prestige ${ }^{1}$, mais surtout, il multiplie à l'occasion des meetings les prises de distance par rapport au support écrit. Certes, il lit : mais toutes les occasions sont bonnes pour s'éloigner du pupitre, changer de registre de langue, glisser de l'analyse à l'anecdote ${ }^{2}$. Ces écarts manifestent clairement l'intrusion de l'individu-candidat dans un discours que chacun sait produit par un collectif institutionnel. Nicolas Sarkozy a dans ces conditions d'autant moins de scrupules à médiatiser celui qui est sa plume (Henri Guaino) qu'il n'est jamais prisonnier du travail de ce dernier. Le candidat s'affirme aussi (et peut-être d'abord) dans sa capacité à faire exister un je individualisé, plus authentique que stratégique, plus personnel qu'institutionnel. Le discours de ce je est évidemment chargé d'émotions et d'affects. La digression est prétexte à bons mots pour faire rire, elle est aussi le temps de l'indignation lorsqu'il faut oser appeler un chat un chat.

Si le cas de Nicolas Sarkozy est idéal-typique, le phénomène dont il témoigne est évidemment beaucoup plus large. La multiplication des contextes de communication non institutionnels a rendu possible, voire normal, le rapport relâché à la langue légitime. L'exemple le plus évident est constitué par Tweeter : si certains s'offusquent encore des libertés prises par les politiques empruntant cette forme de communication, les intéressés ont beau jeu de répondre qu'ils ne font ici que s'ajuster

1 Mentionnons les principaux : Libre (2001), Témoignage (2006), Ensemble (2007).

2 Voir par exemple le discours de Villepinte (11 mars 2012). 
au monde dans lequel ils vivent et avec lequel ils se doivent d'être en phase.

On pourrait de même évoquer les nouveaux formats de la télévision politique, en particulier les talk-shows (Leroux, Riutort, 2013) : les émissions politiques traditionnelles attestaient d'une présence encore massive des institutions. Longtemps les journalistes se sont déplacés pour aller au-devant de celles-ci. Lorsque la technologie a rendu l'exercice délicat, ce sont les politiques qui ont appris à se déplacer. Mais dans un premier temps les plateaux de télévision pouvaient encore apparaître comme des lieux politiques à part entière : présence de journalistes politiques, questionnements exclusivement politiques, labellisation de l'émission comme politique. Ce n'est que récemment que les choses ont changé dans le sens d'une désinstitutionnalisation. Quand ils s'expriment dans les multiples talk-shows qui font désormais l'ordinaire de la télévision grand public, les politiques sont jugés sur leur capacité à endosser le rôle d'individu et à se libérer des rôles institutionnels incompatibles avec la société de spectacle. Il leur faut faire la preuve de leur capacité à se " décoincer ", se " décrisper ", se « libérer » des habitus institutionnels. Savoir rire (y compris de soi et de son moi institutionnel), savoir sortir de son rôle, savoir changer de registre, apparaître à contre-emploi : la légitimité médiatique se construit clairement aux dépens de la légitimité institutionnelle. Il va sans dire que dans ce contexte le langage utilisé ne saurait être celui des institutions. On attend des politiques qu'il se lâchent.

\section{L'optimum de relâchement discursif}

Les remarques précédentes pourraient laisser penser qu'on est passé d'un mode de production discursive à l'autre : obligation de respecter la norme linguistique avant, possibilité de s'en écarter aujourd'hui... En réalité, les choses ont évolué de façon plus subtile : les deux modes de production cohabitent, et il appartient aux politique de solliciter alternativement l'un et l'autre registre selon les contextes et les situations. L'exemplarité linguistique demeure la norme dans les contextes strictement institutionnels, en l'absence de médias (conseil des ministres) ou lorsque ces derniers s'inclinent devant le format institutionnel (débats parlementaires). L'écart par rapport à la norme est possible, voire souhaité, dans les médias les plus déconnectés des institutions (talk-shows) : on peut alors se demander ici si le relâchement ne constitue pas une norme aussi contraignante que les précédentes. Il 
est sans doute plus difficile aux énarques d'origine bourgeoise de se convertir au ton relâché des plateaux de télévision que d'emprunter à la langue académique des institutions. Et le «naturel » situe à l'évidence certains d'entre eux davantage du côté des institutions que du côté des médias. Entre ces deux pôles, il existe évidemment toute une série de situations intermédiaires : lorsque les institutions sont médiatisées (questions au gouvernement, conférences de presse), lorsque les médias s'institutionnalisent (émissions dite politiques, soirées électorales)... Il faut alors savoir trouver un juste milieu.

Ces remarques suggèrent qu'une des qualités aujourd'hui nécessaires à l'endossement du rôle de personnalité politique tient dans la capacité à bien saisir la situation et à alterner les registres. Édouard Balladur hier, Dominique de Villepin plus récemment, ont été moqués pour leur incapacité à s'extraire d'une allure de grand bourgeois ou d'aristocrate. Leur présidentiabilité s'en est trouvée affectée. A l'inverse, les critiques adressées à Nicolas Sarkozy ont souvent visé un comportement jugé en deçà des exigences de la fonction présidentielle. L'optimum de relâchement est à l'évidence difficile à atteindre. S'il faut savoir prendre des libertés par rapport à la norme la plus rigide, il faut aussi savoir jusqu'où ne pas aller trop loin (Wouters, 2012). Il est ici frappant de constater l'émotion suscitée par les gaffes et autres écarts de langage des politiques en situation institutionnelle (Le Bart \& Teillet, 2004). Les journalistes ne laissent rien passer, les commentateurs reprennent en boucle, les internautes s'indignent... Tout le monde a en tête le procès en incompétence que valut à Ségolène Royal le néologisme «bravitude » lors de la campagne de 2007. Le procès en illégitimité vise particulièrement les entrants faiblement pourvus en ressources académiques et politiques. Le jour même de sa nomination comme ministre (1 juillet 2011), l'ancien judoka David Douillet se faisait ainsi épingler sur Europe 1 par le journaliste Benjamin Muller. Reprenant le titre de France-Soir : (« David Douillet : premier jour et première gaffe »), celui-ci déclarait : «Si on veut être sympa, on peut dire que sa langue a fourché ; sinon, ben sinon, on n'a pas d'excuse pour lui » (Benjamin Muller). La faute du nouveau ministre ? Avoir commis un lapsus (« tous les domaines possibles et inimaginaux» au lieu de imaginables). Et l'éditorialiste de poursuivre : « Est-ce grave cette ligne Maginot de domaines inimaginables ? Parce que franchement depuis ce matin ça n'arrête pas sur le Net. Chacun y va de sa vanne contre le pauvre David Douillet. Mais qu'il se rassure, notre ministre, il n'est pas le seul, loin de là... ». 
Ces exemples suggèrent la permanence d'une exigence d'exemplarité linguistique de la part des candidats. Cette norme n'est pas seulement active à l'intérieur des institutions. Elle participe des attentes des citoyens à l'endroit des politiques. La capacité des internautes à réagir sur ces questions, quoique difficile à mesurer, est réelle. Attention toutefois à ne pas confondre opinion publique et leaders d'opinion : l'indignation des médias ne dit rien des attentes réelles des citoyens, comme le suggère l'exemple de Rachida Dati, brocardée pour des écarts malheureux mais durablement installée parmi les personnalités politiques préférées des Français.

Cette norme d'exemplarité persiste tout particulièrement au sommet de l'État. Comme Georges Bush aux Etats-Unis, comme Berlusconi en Italie, Nicolas Sarkozy a sans doute sous-estimé l'exigence persistante d'exemplarité à l'endroit de quiconque endosse la fonction exécutive la plus élevée. En parlant de " présidence normale », François Hollande entendit rappeler ce devoir d'exemplarité. En sens inverse, l'absence totale de fantaisie discursive, le respect scrupuleux des normes et des rôles, peut être dénoncée comme non conforme aux lois du spectacle. La présidence normale de François Hollande pêche peut-être, au même titre que le style de Jean-Marc Ayrault, par manque de charisme. L'ancrage institutionnel revendiqué par l'un et l'autre néglige sans doute l'ancrage médiatique.

\section{Références}

Bourdieu, P., \& Passeron, J.-C. (1970). La reproduction. Paris : Minuit.

Charaudeau, P. (2008). Entre populisme et peopolisme : comment Sarkozy a gagné ! Paris : Vuibert.

Dubet, F. (2002). Le déclin de l'institution. Paris : Seuil.

Le Bart, C. (1998). Le discours politique. Paris : PUF.

Le Bart, C., \& Teillet P. (2004). Erreurs, lapsus, gaffes, fautes... : le discours politique comme genre. Dans R. Ringoot \& P. Robert-Demontrond (Éd.), L'analyse $d u$ discours (pp. 53-86). Rennes : Apogée.

Leroux, P., \& Riutort, P. (2013). La politique sur un plateau. Paris : PUF.

Mayaffre, D. (2004). Paroles de président. Jacques Chirac et le discours présidentiel sous la Vème République. Paris : Champion.

Mayaffre, D. (2010). Trente ans d'étude des langages politiques (1980-2010). Mots, 94. Rousselier, N. (1997). Le parlement de l'éloquence. Paris : Presses de la FNSP.

Wouters, C. (2012). Comment les processus de civilisation se sont-ils prolongés ? De la 'seconde nature' à la 'troisième nature'. Dans Q. Deluermoz (Éd.), Norbert Elias (pp. 331-360). Paris : Perrin. 